\title{
PERSONAL FABLES, NARCISSISM, AND ADOLESCENT ADJUSTMENT
}

\author{
MATTHEW C. AALSMA \\ Indiana University School of Medicine \\ DANIEL K. LAPSLEY \\ Ball State University \\ DANIEL J. FLANNERY \\ Kent State University and University Hospitals of Cleveland
}

\begin{abstract}
The relationship among three personal fables (omnipotence, invulnerability, personal uniqueness), narcissism, and mental health variables was assessed in a large, cross-sectional sample of adolescents drawn from Grades $6(n=94), 8(n=223), 10(n=142)$, and $12(n=102)$. Participants responded to the New Personal Fable Scale, the Narcissistic Personality Inventory, the Children's Depression Inventory, three indices of suicidal ideation, an inventory of delinquent risk behaviors, the Global Self-Worth scales from the Self-Perception Profiles for Children and for Adolescents, and two subscales from the Self-Image Questionnaire for Young Adolescents. The results showed that omnipotence and narcissism strongly counterindicated internalizing symptomatology, and were robust predictors of positive mental health and adjustment. Invulnerability was strongly associated with risk behaviors. Personal uniqueness was strongly associated with depression and suicidal ideation, a relationship that increased with age. Hence, personal fable ideation is a multidimensional construct with differential implications for adolescent mental health. Adolescent fables of invulnerability and personal uniqueness are risk factors for externalizing and internalizing symptoms, respectively, while "narcissistic omnipotence" is associated with competence. Implications for theory, practice, and future research are discussed. (c) 2006 Wiley Periodicals, Inc.
\end{abstract}

A significant minority of teenagers report lifetime incidence of clinically relevant symptomatology serious enough to warrant the attention of mental health professionals (Roberts, Attkission, \& Rosenblatt, 1998). These difficulties are rarely a "passing phase" that adolescents outgrow. Instead, symptom formation in adolescence reflects deviant adjustment and psychopathology (Weiner, 1992). Moreover, these disturbances exert a persistent deleterious influence that adversely affects the mental health of young people well into adulthood (Fleming, Boyle, \& Offord, 1993; Kandel \& Davies, 1986). Consequently, evidence of mental health disturbances in adolescence rightly commands the attention of school personnel, counselors, and researchers.

Within the past two decades, a developmental approach to psychopathology has emerged for conceptualizing and investigating variations in competence, vulnerability, and maladaptation. This approach suggests that the study of normative developmental processes provides a valuable framework for understanding atypical or psychopathological behavioral patterns. In this case, maladaptive behavior is understood not simply in terms of manifest clinical symptomatology but also in terms of deviation from normal development or as a corruption of normative developmental processes (Sroufe \& Rutter, 1984). Moreover, patterns of adaptation may well show important agedependent variations. If true, then the search for causal antecedents of adaptational failures might well focus on the developmental challenges posed by particular age periods, investigated on its own terms, as well as on developmental precursors from early life. As Sroufe and Rutter (1984) noted, "we would do well to categorize patterns of adaptation (and maladaptation) in terms of the

Correspondence to: Daniel K. Lapsley, Department of Educational Psychology, Teachers College 520, Ball State University, Muncie, IN 47306. E-mail: dklapsley@bsu.edu 
child's management of salient age-period issues" (p. 24). Of course, how these salient, age-defined issues and normative age-related milestones are conceptualized depends heavily on the adequacy of developmental theory.

In the present study, our conceptualization of the salient issues of this developmental period was guided by two constructs that bear some theoretical resemblance. The first is the well-known notion, first suggested by Elkind (1967), that adolescents construct personal fables. The second notion is the claim, often found in the psychodynamic literature (e.g., Blos, 1962), that teenagers are particularly disposed to narcissistic displays, perhaps as a defensive maneuver to cope with various aspects of personality development during adolescence. Our aim was to determine how personal fable and narcissistic ideation are related with adolescent mental health and adjustment.

\section{Personal FABLe}

According to Elkind (1967), adolescents construct personal fables as one consequence of their cognitive egocentrism. The content of adolescent personal fables typically includes themes of invulnerability (i.e., an incapability of being harmed or injured), omnipotence (i.e., viewing the self as a source of special authority or influence), and personal uniqueness (i.e., "No one understands me"). This theory has been invoked as a framework for understanding the developmental components of faulty risk perception and risk-taking behavior (Arnett, 1992). An alternative conceptualization views the personal fable not as an outcome of cognitive egocentrism but rather as an adaptive response to the demands of adolescent ego development (Lapsley, 1993; Lapsley \& Rice, 1988). According to this view, an inflated sense of personal uniqueness, omnipotence, and invulnerability is an attempt by the adolescent to maintain the boundaries, integrity, and cohesiveness of the self as the adolescent wrestles with the second phase of separation-individuation (e.g., Blos, 1962). This described personal fable ideation is identical to the narcissistic restitution strategy described by Blos (1962), whereby the adolescent's newly keen perception of inner life, and his or her "willful creation of ego states of a poignant internal perception of the self" (p. 98), lead to a heightened sense of uniqueness, indestructibility, and personal agency.

Yet, a more positive conceptualization of personal fable ideation is possible. Bjorklund and Green (1992) argued, for example, that personal fable tendencies may facilitate the taking of appropriate risks, motivate psychological separation from parents, and provide the resources for adolescents to explore new ideas, identities, roles, and tasks. Cole (1989) suggested that personal fable ideation may be a cognitive factor that attenuates the effects of hopelessness in adolescence, thereby serving as a buffer between hopelessness and suicidal behavior. Finally, there is evidence to suggest that personal fable ideation may serve adaptive outcomes in the context of separation-individuation (Goossens, Beyers, Emmen, \& van Aken, 2002; Lapsley, FitzGerald, Rice, \& Jackson, 1989).

In the present study, we expanded our look at these questions by examining the relationship between personal fable ideation and a much wider range of mental health assessments than is currently available in the literature. One set of measures tapped internalizing tendencies toward depressive symptomatology and suicidal ideation. A second set examined adolescents' involvement in a variety of risk behaviors such as vandalism, stealing, and driving fast as well as the use of drugs, alcohol, and tobacco. We hypothesized that personal fable ideation might constitute a risk factor for externalizing behaviors, but would otherwise counterindicate depressive affect and suicidal tendencies. A third battery of measures assessed positive aspects of adolescent functioning. It is conceivable that adolescents who feel omnipotent, invulnerable, or personally unique might well report very few internalizing symptoms, but whether these fables also are associated with psychosocial competence is another matter. 


\section{NARCISSISM}

Narcissistic grandiosity, exhibitionism, and heightened self-consciousness are thought to be commonplace in adolescents; however, although narcissism is often attributed to teenagers, empirical studies of adolescent narcissism and its consequences for mental health are vanishingly rare (Lapsley \& Aalsma, in press). It has been suggested that exaggerated personal fable tendencies might well converge with diagnostic descriptions of narcissistic personality (Lapsley \& Rice, 1988). We included a measure of narcissism in the present study to chart its relationship with a broader range of mental health and adjustment indices than has been reported in the literature thus far, particularly in samples of adolescents. Given its theoretical similarity with personal fable ideation, we expected the two constructs to perform similarly with respect to internalizing and externalizing tendencies in adolescents and in terms of positive adjustment.

\section{METHOD}

\section{Participants}

Participants included 94 sixth graders, 223 eighth graders, 142 tenth graders, and 102 twelfth graders, for a sample total of 561 participants. The 6th-grade sample (50 males, 41 females; 3 did not report gender and are treated as missing data for all analyses involving gender) had a mean age of 11.44 years $(S D=.62)$. The 8th-grade sample (101 males, 93 females; 29 did not report gender and are similarly treated as missing data) had a mean age of 13.39 years $(S D=$ $.56)$. The 10th-grade sample ( 72 males, 70 females) had a mean age of 15.43 years $(S D=.66)$. The 12th-grade sample (46 males, 56 females) had a mean age of 17.45 years $(S D=.71)$. These participants lived in a small city in Western Canada. The schools sampled in this study tended to reflect the diversity of student characteristics for this community, being largely Caucasian and lower middle to middle class.

\section{Instruments and Procedure}

Personal fable. The New Personal Fable Scale (NPFS; Lapsley et al., 1989) includes Likerttype scales that assess a belief in personal uniqueness (13 items; $\alpha=.69$ ), invulnerability (14 items; $\alpha=.72$ ), and omnipotence (19 items; $\alpha=.79$ ). Psychometric properties of the NPFS have been generally adequate (Goossens et al., 2002; Vartanian, 1997).

Narcissism. The Narcissistic Personality Inventory (NPI; Raskin \& Hall, 1981) measures individual differences in narcissism as it is expressed in nonclinical populations. It consists of 40 items, each of which is paired with a more neutral statement. Participants must select the one statement within each pair with which they most agree. The NPI appears to have strong psychometric properties (e.g., Raskin, Novacek, \& Hogan, 1991). The KR-20 estimate of internal consistency in the present study was .83.

Internalizing symptoms. Depressive symptomatology was assessed by the Children's Depression Inventory (CDI; Kovacs, 1985; $\alpha=.91$ ). Suicidal ideation was assessed by culling relevant items from three standard measures of depression and suicidal behavior. Three items were selected from the Suicidal Behaviors Questionnaire (SBQ; Linehan \& Nielsen, 1981; $\alpha=.85$ ), four items were used from the Zung Index of Potential Suicide (ZIPS; Zung, 1965, $\alpha=.81$ ), three items were culled from the Center for Epidemiological Studies of Depression Scale (CES-D; Radloff, 1977; $\alpha=.86$ ).

Risk behaviors. Risk-taking tendencies were assessed by a self-report delinquency battery developed by Rowe $(1985 ; \alpha=.92)$. Participants were asked to rate the extent to which they engaged in a list of 20 risk behaviors, including fast driving, stealing, fighting, and vandalism. In addition, participants also were asked to indicate their lifetime usage $(\alpha=.74)$ and frequency of 
usage ( $\alpha=.73$ ) of alcohol, drugs (inhalants, marijuana, hallucinogens, cocaine/crack, amphetamines), and tobacco.

Positive adjustment and mental health. Adaptive aspects of adolescent functioning was assessed by a measure of global self-worth derived from Harter's (1982) Self-Perception Profile for Children (Grades $6 \& 8, \alpha=.83$ ) and for Adolescents (Grades $10 \& 12, \alpha=.70$ ), and by the mastery and coping $(\alpha=.76)$ and superior adjustment $(\alpha=.70)$ subscales from the Self-Image Questionnaire for Young Adolescents (SIQYA; Petersen, Schulenberg, Abramowitz, Offer, \& Jarcho, 1984).

\section{RESULTS}

Grade (4) $\times$ Gender (2) MANOVAs were calculated to assess group differences among three sets of variables measured in this study. The first MANOVA included narcissism and the three personal fable measures (i.e., omnipotence, invulnerability, personal uniqueness) as dependent variables. A significant multivariate effect was observed for grade, Pillai $=.07, F=2.81, p=.001$, and for gender, Pillai $=.18, F=23.47, p=.00$. The multivariate interaction was not significant. Univariate tests showed that the grade effect was limited to the variable invulnerability, $F(3,441)=$ $5.18, p=.002$. Post hoc tests with the Scheffe procedure showed that both 8 th graders and 10th graders were more invulnerable than 6th graders. Univariate analysis of the multivariate gender effect revealed significant gender differences for omnipotence, $F(1,441)=44.72, p=.00$, for invulnerability, $F(1,441)=67.53, p=.00$; for personal uniqueness, $F(1,441)=9.57, p=.02$, and for narcissism, $F(1,441)=31.73, p=.00$. Males reported more omnipotence, invulnerability, and narcissism. Females reported a greater sense of personal uniqueness.

The second Grade $\times$ Gender MANOVA included a linear combination of the "externalizing" variables (i.e., delinquent risk behaviors and lifetime drug use and frequency) as the dependent variables. The results indicated a significant multivariate effect for grade, Pillai $=.25, F=14.62$, $p=.00$, and for gender, Pillai $=.16, F=30.71, p=.00$. The multivariate interaction was not significant. Univariate tests showed that the significant grade effect was accounted for by delinquent risk behaviors, $F(3,496)=7.90, p=.00$, by lifetime drug use, $F(3,496)=39.56, p=.00$, and by frequency of drug use, $F(3,496)=24.29$. Post hoc analyses using the Scheffe procedure indicated that 10th and 12th graders reported a greater frequency of drug use than did 6th graders, and 12th graders more than 8th graders. Regarding lifetime drug use, 10th graders reported more than did 6th and 8th graders; 12th graders also reported more lifetime drug use than did 6th and 8 th graders. The multivariate gender effect was accounted for by gender differences on delinquent risk behaviors, $F(1,496)=68.38$, with males reporting higher scores than females.

The third Grade $\times$ Gender MANOVA included the internalizing symptoms (i.e., depression and the measures of suicidal ideation) as dependent variables. A significant multivariate effect emerged for grade, Pillai $=.07, F=3.06, p=.00$, and for gender, Pillai $=.035, F=4.36, p=.00$. The Grade $\times$ Gender interaction was not significant. Univariate analysis of the grade effect showed significant grade differences for depression, $F(3,484)=4.01, p=.01$, and for suicidal ideation (ZIPS), $F(3,484)=3.12, p=.01$. Post hoc analysis showed that 8 th and 10 th graders reported more depressive symptoms than did 12 th graders, and that 10 th graders reported more suicidal ideation than did 12 th graders. Univariate gender differences emerged for depression, $F(1,484)=$ $11.07, p=.001$, for suicidal ideation (ZIPS), $F(1,484)=8.35, p<.00$, for suicidal ideation $(\mathrm{SBQ}), F(1,484)=16.08, p<.00$, and for suicidal ideation $(\mathrm{CES}-\mathrm{D}), F(1,484)=9.37, p=.002$. In all cases, females reported more internalizing symptoms than did males.

A multivariate analysis of the fourth set of variables comprising positive adjustment (i.e., self-worth, mastery/coping, superior adjustment) was not possible insofar as younger and older 
adolescents responded to different versions of the Self-Perception Profile (self-worth). Hence, group differences for these variables were assessed with univariate Grade $\times$ Gender ANOVAs. A Grade (4) $\times$ Gender (2) analysis of mastery/coping revealed a significant effect for gender, $F(1,510)=20.25, p<.00$, favoring males (i.e., males indicating better mastery/coping). The main effect for grade and the interaction term were not statistically significant. The analysis of superior adjustment revealed significant main effects for gender, $F(1,507)=4.57, p=.03$, favoring males, and for grade, $F(3,507)=6.24, p<.00$. Post hoc analysis using the Scheffe procedure showed that 6 th graders were better adjusted than were 8 th, 10th, and 12th graders. The interaction term was not statistically significant.

The Grade (2) $\times$ Gender (2) analysis of global self-worth among 6th and 8th graders revealed no significant effects; however, a significant gender effect did emerge in the self-worth analysis of 10th and 12th graders, $F(1,233)=11.61, p=.001$, favoring males, although the slight mean difference would appear to be of little practical value.

\section{Correlational Analyses}

The pattern of correlation among the variables, partialing age, is reported in Table 1. With respect to the internalizing variables, omnipotence was negatively correlated with depression $(r=$ $-.52)$ and with the three indices of suicidal ideation $\left(M_{\mathrm{r}}=-.33\right)$. Invulnerability was unrelated to suicidal ideation, and only modestly counterindicated depression $(r=-.13)$. In contrast, the fable of personal uniqueness was positively correlated with depression $(r=.33)$ and with each of the indices of suicidal ideation $\left(M_{\mathrm{r}}=.28\right)$.

A similar pattern emerges with respect to positive adjustment. Omnipotence was strongly correlated with mastery and coping $(r=.57)$, with superior adjustment $(r=.58)$, and with the two

Table 1

Partial Correlations Among Indices of Personal Fable Ideation, Narcissism, and Adjustment*

\begin{tabular}{|c|c|c|c|c|}
\hline Adjustment variables & Omnipotence & $\begin{array}{l}\text { Personal fables } \\
\text { invulnerability }\end{array}$ & Uniqueness & Narcissism \\
\hline \multicolumn{5}{|l|}{ Externalizing } \\
\hline Risk behaviors & .09 & .44 & .03 & .24 \\
\hline Drug Frequency & -.14 & .21 & .09 & .04 \\
\hline Lifetime drug use & -.09 & .23 & .10 & .11 \\
\hline \multicolumn{5}{|l|}{ Internalizing } \\
\hline Depression & -.52 & -.13 & .33 & -.29 \\
\hline Suicidal ideation (ZIPS) & -.27 & -.01 & .27 & -.10 \\
\hline Suicidal ideation (CES-D) & -.37 & -.07 & .25 & -.20 \\
\hline Suicidal ideation (SB-Q) & -.34 & -.02 & .30 & -.12 \\
\hline \multicolumn{5}{|l|}{ Positive Adjustment } \\
\hline Mastery coping & .57 & .29 & -.23 & .44 \\
\hline Superior adjustment & .58 & .17 & -.12 & .47 \\
\hline Self-worth (6-8th grades) & .55 & .16 & -.23 & .34 \\
\hline Self-worth (10-12th grades) & .58 & .26 & -.34 & .40 \\
\hline Narcissism & .67 & .47 & -.03 & - \\
\hline
\end{tabular}

*Bolded correlations indicate significant at $p<.05$. 
measures of global self-worth $\left(M_{\mathrm{r}}=.56\right)$. The magnitude of the relationship between invulnerability and these variables is attenuated, although statistically significant and in the same direction. In contrast, personal uniqueness was associated with poorer mastery/coping $(r=-.23)$, poorer adjustment $(r=-.12)$, and lower self-worth $\left(M_{\mathrm{r}}=-.29\right)$.

A somewhat different pattern emerged with respect to delinquent risk behaviors and substance use. As one might expect, the sense of invulnerability was strongly correlated with delinquent risk behaviors $(r=.44)$ and with the use of various substances $\left(M_{\mathrm{r}}=.22\right)$. But omnipotence was virtually uncorrelated with delinquent risk and was negatively correlated, albeit modestly, with the frequency of drug use $(r=-.14)$. Similarly, personal uniqueness was largely uncorrelated with delinquent risk, although it was modestly correlated with lifetime incidence of substance use $(r=.10)$.

It would appear, then, that omnipotence and, to a lesser extent, invulnerability, counterindicated depression and suicidal ideation and was positively associated with better coping, adjustment, and self-worth. In contrast, the fable of personal uniqueness was positively associated with more depressive symptoms, more suicidal ideation, and with a poorer profile of coping, adjustment, and self-worth. On the externalizing side, invulnerability was a stronger predictor of risk behaviors and drug use than were the other personal fables.

Note that narcissism was similar to omnipotence $(r=.67)$ in its profile of correlations with internalizing symptoms and with indices of positive mental health, and similar to invulnerability $(r=.47)$ in its profile of correlations with risk behaviors. Hence, much like omnipotence, narcissism was negatively correlated with the internalizing symptoms depression $(r=-.29)$ and suicidal ideation $\left(M_{\mathrm{r}}=-.14\right)$, and positively associated with superior adjustment, mastery coping, and self-worth $\left(M_{\mathrm{r}}=.41\right)$; however, much like invulnerability, narcissism also predicted engagement with risk behaviors as well $(r=.24)$.

Analysis of correlational trend. Next, we determined if the pattern of correlations just noted would show age-related variation from early to late adolescence. To this end, a correlation matrix was generated within each grade. The correlation coefficients were then standardized (via Fisher's $r-z$ transformation), and analyzed for linear and quadratic trend (where indicated) across grade. To keep the number of analyses to an informative minimum, we restricted our search for developmental functions to a smaller set of questions that are suggested by the consistent pattern of correlations observed earlier with the personal fable variables.

The results are summarized in Table 2. There was a significant linear increase from early to late adolescence in the magnitude of the correlation between personal uniqueness and depressive symptoms, and between personal uniqueness and each of the three indices of suicidal ideation. Moreover, there was a significant quadratic component, which was accounted for by the somewhat attenuated relationship between personal uniqueness and the internalizing variables in Grade 12 . No significant developmental variations were evident in the relationship between invulnerability and risk behaviors; it was consistently moderate at each grade level. Regarding omnipotence, the relationship between omnipotence and mastery/coping was significantly stronger from early to late adolescence. Moreover, the significant quadratic trend suggested that the negative relationship between omnipotence and depression is particularly pronounced in middle adolescence. Finally, no significant developmental variations were evident in the relationship between narcissism and risk behaviors, depression, and positive adjustment, respectively.

\section{Discussion}

The purpose of this study was to examine the role of personal fable ideation and narcissism in adolescent mental health. Although there is a general consensus that both kinds of ideation are 
Table 2

Summary of Correlation Coefficients by Grade and Z Tests of Linear and Quadratic Tests of Correlational Trend*

\begin{tabular}{|c|c|c|c|c|c|c|}
\hline & \multicolumn{6}{|c|}{ Grade } \\
\hline & 6 & 8 & 10 & 12 & Linear & Quadratic \\
\hline \multicolumn{7}{|l|}{ Personal uniqueness } \\
\hline$r$ with depression & -0.01 & 0.40 & 0.48 & 0.29 & 2.13 & 3.16 \\
\hline$r$ with suicidal (SBQ) & 0.05 & 0.33 & 0.51 & 0.39 & 2.67 & 2.22 \\
\hline$r$ with suicidal (CES-D) & -0.09 & 0.33 & 0.42 & 0.34 & 3.07 & 2.78 \\
\hline$r$ with suicidal (ZIPS) & -0.08 & 0.32 & 0.39 & 0.33 & 3.11 & 3.11 \\
\hline \multicolumn{7}{|l|}{ Invulnerability } \\
\hline$r$ with risk behavior & 0.29 & 0.46 & 0.43 & 0.51 & 1.40 & not tested \\
\hline$r$ with drug frequency & 0.15 & 0.17 & 0.27 & 0.24 & 0.80 & not tested \\
\hline$r$ with lifetime drug use & 0.14 & 0.20 & 0.25 & 0.25 & 0.83 & not tested \\
\hline \multicolumn{7}{|l|}{ Omnipotence } \\
\hline$r$ with mastery coping & 0.49 & 0.58 & 0.65 & 0.72 & 1.69 & not tested \\
\hline$r$ with superior adjustment & 0.59 & 0.60 & 0.54 & 0.65 & -0.27 & not tested \\
\hline$r$ with depression & -.43 & -.65 & -.59 & -.42 & .10 & 2.17 \\
\hline$r$ with suicidal (SBQ) & -.29 & -.36 & -.25 & -.43 & -.67 & -.61 \\
\hline$r$ with suicidal (CES-D) & -.22 & -.46 & -.29 & -.47 & -1.26 & .33 \\
\hline$r$ with suicidal (ZIPS) & -.25 & -.33 & -.20 & -.40 & -.69 & -.67 \\
\hline \multicolumn{7}{|l|}{ Narcissism } \\
\hline$r$ with risk behavior & .15 & .18 & .21 & .38 & 1.53 & not tested \\
\hline$r$ with depression & -.31 & -.33 & -.33 & -.20 & .70 & not tested \\
\hline$r$ with mastery coping & -.39 & -.41 & -.49 & -.59 & 1.45 & not tested \\
\hline$r$ with superior adjustment & -.49 & -.55 & -.41 & -.49 & .29 & not tested \\
\hline
\end{tabular}

*Bolded $z$ tests indicate significant at $p<.05$.

commonplace and normative during adolescence, there is far less agreement on what their respective implications are for adaptive functioning.

The analysis of grade effects showed that narcissism and the fables of omnipotence and personal uniqueness did not show significant variation from early to late adolescence, although a significant age-related increase in invulnerability was evident from early (6th grade) to middle (8-10th grades) adolescence. Depressive affect and suicidal ideation also tended to rise from early to middle adolescence-findings that also have been reported in the literature.

A number of significant gender differences also were observed in the present study. As predicted, males were more narcissistic than females. Males also were more invulnerable and omnipotent, and engaged in more risk behaviors than did females. In contrast, females reported more depressive symptoms, more suicidal ideation, and greater feelings of personal uniqueness than did males. The greater tendency of males to be narcissistic and to endorse the "agentic" qualities of personal fable ideation (i.e., invulnerability, omnipotence) replicates previous work in this area (Lapsley et al., 1989). Moreover, the fact that males report a higher involvement with risk behaviors and that females report more depressive affect also is congruent with the well-known preference of males for externalizing coping strategies and of females for internalizing strategies. Hence, there is an encouraging degree of convergence between the age and gender differences reported here and general trends reported in the literature. 
Correlational analyses, including tests of correlational trend, indicated that the "omnipotence" fable was consistently associated with positive adaptational outcomes. It was a strong predictor of self-worth, mastery and coping, and superior adjustment. Indeed, the correlation between omnipotence and mastery/coping was significantly stronger with age. In addition, omnipotence strongly counterindicated depressive affect and suicidal ideation.

In contrast, the personal uniqueness fable has a uniformly negative association with mental health, and virtually no redeeming qualities. Most notably, this fable was a powerful predictor of depressive mood and of suicidal ideation, an association that got stronger from early to middle adolescence. Hence, personal uniqueness is an apparent risk factor for internalizing symptomatology, and the risk it carries for depression and suicidal ideation becomes increasingly more significant with age. A similar finding also was reported by Goossens et al. (2002).

Invulnerability was a significant predictor of a variety of risk behaviors, including the use of tobacco, beer, and other controlled substances; however, although there was a significant increase in reported invulnerability between early to middle adolescence, there was no developmental variation in the bivariate relationship between invulnerability and risk behaviors. Indeed, the correlation between invulnerability and risk behaviors was consistently moderate in all four grades sampled here. The stability of this relationship from early to late adolescence leads one to suspect that it also might hold in adulthood as well, which cautions against the presumption of the singularly invulnerable adolescent (Millstein \& Halpern-Felsher, 2001, 2002). Note also that invulnerability did not operate solely as a risk factor in these data. Invulnerability also predicted, albeit less strongly, certain features of positive mental health such as self-worth, mastery coping, and superior adjustment.

Similarly, narcissism also was associated with both positive and negative aspects of adolescent functioning. Given its strong correlation with omnipotence, it is not surprising that narcissism should show a similar profile of positive correlations with indices of adjustment and negative correlations with internalizing symptoms. Similarly, narcissism also predicted risk behavior and was moderately correlated with invulnerability. Hence, it would appear that narcissism is coextensive with both the omnipotence and invulnerability fables, which in turn have differential implications for adolescent mental health and adjustment.

The fact that narcissistic omnipotence and invulnerability predicted numerous aspects of positive adjustment is congruent with theoretical claims that these are defensive or compensatory mechanisms that support adaptive functioning as the adolescent wrestles with normative developmental challenges (e.g., Blos, 1962).

Of course, narcissistic invulnerability predicted delinquent risk behavior as well. That "narcissistic invulnerability" also should be positively associated with risk behaviors is perhaps not surprising in light of Jessor's (1992) observation that engaging in risk behaviors may well serve normal psychosocial goals in adolescence such as consolidating peer acceptance, affirming maturity and independence, and warding off stress, frustration, and anxiety. Hence, in his view, such behaviors are "functional, purposive, instrumental and goal-directed, and that the goals involved are often those that are central in normal adolescent development" (Jessor, 1992, p. 378), including individuation from parents, experimentation with values, roles, and identities, and the press toward autonomy and independence (Chassin, Presson, \& Sherman, 1989). Although both risk behavior and adjustment are predicted by narcissistic invulnerability, perhaps these apparently divergent relationships point nonetheless to similar developmental ends.

The present study has implications for developmental theory and for psychological practice in the schools. First, the relative complexity of these results makes clear that personal fable ideation is a plural concept, although there has been a tendency in previous research to treat the personal fable as if it were a unidimensional construct with straightforward implications (invariably 
negative) for adolescent mental health. In addition, the fact that narcissism is moderately correlated with two personal fables (i.e., invulnerability and omnipotence) that have such different implications for mental health would seem to inform the ongoing debate about its developmental origins. The traditional view has suggested that personal fable ideation is the result of a differentiation failure ("egocentrism") that accompanies the onset of early formal operations (Arnett, 1992; Elkind, 1967). An alternative view has linked personal fable ideation to the increase in narcissism that is thought to accompany separation-individuation (Lapsley, 1993; Lapsley \& Aalsma, in press). Although the present study was not designed as a critical test of the competing views, it does offer evidence that is at least compatible with a "narcissism" reading of personal fable ideation.

Finally, the present data alert school psychologists and educators to the differentiated outcomes associated with adolescent narcissism and personal fable ideation. The strong positive association between narcissism, omnipotence, and indices of self-worth, coping, and adjustment, along with the strong negative association of narcissistic omnipotence with depressive symptoms and suicidal ideation, suggests that narcissistic tendencies and a sense of omnipotence in adolescence are not invariably evidence of vulnerability or immaturity (Lapsley \& Aalsma, in press). Indeed, these data support an emerging consensus that "the presence of narcissistic tendencies in the personality actively serve to enhance psychological well-being and to promote good feelings about the self" (Davis, Claridge, \& Brewer, 1996, p. 163). Indeed, Aalsma and Lapsley (1999) suggested that the provision of mirroring support should accompany the mobilization of adaptive forms of narcissism as adolescents wrestle with normative developmental challenges.

The present data also suggest that personal uniqueness fables should be treated as a risk factor for internalizing problems, especially in girls. It is well known that a preference for selffocused, ruminative coping strategies is a mechanism that increases risk for depressive symptoms in girls. Perhaps a self-focused preoccupation with one's sense of personal uniqueness is one aspect of what girls ruminate about. Consequently, teenagers who are impressed with the uniqueness of their personal experience might be unwilling to reach out to form the relational alliances that would buffer stress and encourage resilience. They would perhaps not see the point of attempting to form the sort of intimate confiding relationships that could otherwise provide encouragement, support, and guidance - and psychological protection. If no one can possibly understand what I am like, then perhaps it is pointless to make the effort to articulate or share it. But then, such reticence would leave one bereft of the sort of stress-buffering and protective relationships that are associated with psychosocial resilience.

Finally, the present data offer few clues about a proper response to evidence of invulnerability in adolescents. Indeed, the literature is quite conflicted about the onset, duration, and significance of invulnerability in adolescents. For example, research that utilizes a cognitive biases-and-heuristics framework does not find evidence of pronounced adolescent invulnerability compared to adults (e.g., Beyth-Marom, Austin, Fischhoff, Palmgren, \& Jacobs-Quadrel, 1993; Cohn, Macfarlane, Yanez, \& Imai, 1995; Quadrel, Fischhoff, \& Davis, 1993). Researchers who linked health risk behavior to "optimism bias" suggest that increasing perceptions of personal risk and vulnerability would motivate protective behavior (e.g., Brewer, Cuite, Herrington, \& Weinstein, 2004; Weinstein, 1987), although there is evidence that younger adolescents already report pervasive feelings of vulnerability to a greater extent than do young adults (Millstein \& Halpern-Felsher, 2001). Moreover, the present data suggest that while invulnerability predicted both delinquent and health-compromising behaviors, it predicted adaptive functions as well. Consequently, it is by no means clear that undermining an adolescent's sense of invulnerability is desirable if this self-enhancing illusion contributes to psychosocial resilience in the face of normative developmental challenges.

The linkage between perceived invulnerability, risk perception, and risk behavior will need to be explored further in future research. One promising line of research is the exploration of whether 
invulnerability itself is a multidimensional construct with differential implications for intervention (Lapsley, 2003). Future research also should attend to assessment issues. For example, in the present study, invulnerability is understood as a dimension of individual differences; it is an outcome of adolescent personality development. In the cognitive biases-and-heuristics paradigm, invulnerability is inferred by asking individuals to estimate subjective probabilities of negative outcomes (e.g., "zero probability of getting cancer if I smoke"). Attempts to integrate these assessment strategies will pay dividends.

\section{REFERENCES}

Aalsma, M.C., \& Lapsley, D.K. (1999). Religiosity and adolescent narcissism: Implications for values counseling. Counseling and Values, 44, 7-30.

Arnett, J. (1992). Reckless behavior in adolescence: A developmental perspective. Developmental Review, 12, $339-373$.

Beyth-Marom, R., Austin, L., Fischhoff, B., Palmgren, C., \& Jacobs-Quadrel, M. (1993). Perceived consequences of risky behaviors: Adults and adolescents. Developmental Psychology, 29, 549-563.

Bjorklund, D.F., \& Green, B.L. (1992). The adaptive nature of cognitive immaturity. American Psychologist, $47,46-54$.

Blos, P. (1962). On adolescence. New York: Free Press.

Brewer, N.T., Cuite, C., Herrington, J.E., \& Weinstein, N.D. (2004). Risk perceptions and their relation to risk behavior. Annals of Behavioral Medicine, 27, 125-131.

Chassin, L., Presson, C.C., \& Sherman, S. (1989). "Constructive" vs. "destructive" deviance in adolescent health-related behaviors. Journal of Youth and Adolescence, 18, 245-262.

Cohn, L.D., Macfarlane, S., Yanez, C., \& Imai, W.K. (1995). Risk perception: Differences between adolescents and adults. Health Psychology, 14, 217-222.

Cole, D. (1989). Psychopathology of adolescent suicide: Hopelessness, coping beliefs and depression. Journal of Abnormal Psychology, 98, 248-255.

Davis, C., Claridge, G., \& Brewer, H. (1996). The two faces of narcissism: Personality dynamics of body esteem. Journal of Social and Clinical Psychology, 15, 153-166.

Elkind, D. (1967). Egocentrism in adolescence. Child Development, 38, 1025-1034.

Fleming, J.E., Boyle, M.H., \& Offord, D.R. (1993). The outcome of adolescent depression in the Ontario Child Health Study follow-up. Journal of the American Academy of Child and Adolescent Psychiatry, 32, 28-33.

Goldberg, J.H., Halpern-Felsher, B.L., \& Millstein, S.G. (2002). Beyond invulnerability: The importance of benefits in adolescents' decision to drink alcohol. Health Psychology, 21, 477-484.

Goossens, L., Beyers, W., Emmen, M., \& van Aken, M.A.G. (2002). The imaginary audience and personal fable: Factor analyses and concurrent validity of the "new look" measures. Journal of Research on Adolescence, 12, $193-215$.

Harter, S. (1982). The perceived self-competence scale for children. Child Development, 53, 87-89.

Jessor, R. (1992). Risk behavior in adolescence: A psychosocial framework for understanding and action. Developmental Review, 12, 374-390.

Kandel, D.B., \& Davies, M. (1986). Adult sequelae of adolescent depressive symptoms. Archives of General Psychiatry, 43, 255-262.

Kovacs, M. (1985). A children's depression inventory (CDI). Psychopharmacology Bulletin, 21, 995-998.

Lapsley, D.K. (1993). Toward an integrated theory of adolescent ego development: The "new look" at adolescent egocentrism. American Journal of Orthopsychiatry, 63, 562-571.

Lapsley, D.K. (2003). The two faces of adolescent invulnerability. In D. Romer (Ed.), Reducing adolescent risk: Toward an integrated approach (pp. 25-32). Thousand Oaks, CA: Sage.

Lapsley, D.K., \& Aalsma, M.C. (in press). An empirical typology of narcissism and mental health in late adolescence. Journal of Adolescence.

Lapsley, D.K., FitzGerald, D., Rice, K., \& Jackson, S. (1989). Separation-individuation and the "new look" at the imaginary audience and personal fable: A test of an integrative model. Journal of Adolescent Research, 4, 483-505.

Lapsley, D.K., \& Rice, K. (1988). The "new look" at the imaginary audience and personal fable: Toward an integrated model of adolescent ego development. In D.K. Lapsley \& F.C. Power (Eds.), Self, ego, identity: Integrative approaches (pp. 109-129). New York: Springer.

Linehan, M.M., \& Nielsen, S.L. (1981). Assessment of suicidal ideation and parasuicide: Hopelessness and social desirability. Journal of Consulting and Clinical Psychology, 49, 773-775.

Millstein, S.G., \& Halpern-Felsher, B.L. (2001). Perceptions of risk and vulnerability. In B. Fischhoff, E.O. Nightengale, \& J.G. Iannotta (Eds.), Adolescent risk and vulnerability: Concepts and measurement (pp. 15-49). Washington, DC: National Academy Press. 
Millstein, S.G., \& Halper-Felsher, B.L. (2002). Judgments of risk and perceived invulnerability in adolescents and young adulthood. Journal of Research on Adolescence, 12, 399-422.

Petersen, A.C., Schulenberg, J.E., Abramowitz, R.H., Offer, D., \& Jarcho, H.D. (1984). A self-image questionnaire for young adolescents (SIQYA): Reliability and validity studies. Journal of Youth and Adolescence, 13, 93-109.

Quadrel, M.J., Fischhoff, B., \& Davis, W. (1993). Adolescent (in)vulnerability. American Psychologist, 48, $102-116$.

Radloff, L.S. (1977). The CES-D scale: A self-report depression scale for research in the general population. Applied Psychological Measurement, 1, 385-401.

Raskin, R., \& Hall, C.S. (1981). The Narcissistic Personality Inventory: Alternate form reliability and further evidence of construct validity. Journal of Personality Assessment, 45, 159-162.

Raskin, R., Novacek, J., \& Hogan, R. (1991). Narcissism, self-esteem and defensive self-enhancement. Journal of Personality, 59, 19-38.

Roberts, R.E., Attkission, C.C., \& Rosenblatt, A. (1998). Prevalence of psychopathology among children and adolescents. American Journal of Psychiatry, 155, 715-725.

Rowe, D. (1985). Sibling interactions and self-report delinquent behavior: A study of 265 twin pairs. Criminology, 23, 223-240.

Sroufe, A.L., \& Rutter, M. (1984). The domain of developmental psychopathology. Child Development, 55, 17-29.

Vartanian, L. (1997). Separation-individuation, social support and adolescent egocentrism: An exploratory study. Journal of Early Adolescence, 17, 245-270.

Weiner, I.B. (1992). Psychological disturbance in adolescence. New York: John Wiley \& Sons.

Weinstein, N.D. (1987). Unrealistic optimism about susceptibility to health problems: Conclusions from a communitywide sample. Journal of Behavioral Medicine, 10, 481-500.

Zung, W.W.K. (1965). A self-rating depression scale. Archives of General Psychiatry, 12, 63-70. 